\title{
Estimation in self-initiated working memory for spatial locations
}

\author{
Hagit Magen ${ }^{1}$ - Tatiana Aloi Emmanouil ${ }^{2}$
}

Published online: 13 August 2018

(C) Psychonomic Society, Inc. 2018

\begin{abstract}
The short-term maintenance of precise location information is fundamental in many daily activities. Often, individuals memorize spatial information provided to them, but in many other occasions memory is self-initiated, meaning that individuals memorize locations they selected themselves. While prevalent in everyday behavior, research on self-initiated working memory (WM) is scarce. The current study employed a modified spatial precision memory task in which participants temporarily memorized single locations they themselves selected within a square area that appeared during encoding. We explored the distribution of locations participants selected, guided by the assumption that participants would select locations that they perceived would maximize accuracy. The results of two experiments demonstrated that, to enhance memory performance, participants most often selected prototypical locations within the square area, namely, at its center and at its corners, which were closest to the center and the corners of the entire screen. When asked to disrupt memory performance, participants mostly avoided these locations. Furthermore, memory accuracy for self-initiated locations was superior to memory for provided locations, even when the distribution of locations in the two conditions was matched. We interpret the results within the framework of models that emphasize the utility of spatial categories in maximizing memory accuracy in spatial-estimation tasks. The results provide the first illustration of self-initiated representations in spatial precision WM tasks, suggesting that participants have access to metacognitive knowledge about the usefulness of spatial categories in location memory. Moreover, the results demonstrate that spatial precision is enhanced when participants reproduce locations they selected themselves.
\end{abstract}

Keywords Spatial memory $\cdot$ Self-initiation $\cdot$ Visual working memory $\cdot$ Memory biases

Numerous everyday activities require the encoding and short retention of precise visual information. In many instances, the retained information is self-initiated, such as when individuals leave objects in certain locations and retrieve them shortly after. Research on working memory (WM), however, has failed to account for the processes involved in actively shaping the contents of one's own memory. Our main prediction regarding self-initiated working memory (SI WM) was that

Electronic supplementary material The online version of this article (https://doi.org/10.3758/s13423-018-1514-x) contains supplementary material, which is available to authorized users.

Hagit Magen

msmagen@mail.huji.ac.il

1 The Hebrew University, PO Box 24026, Mount Scopus, 91240 Jerusalem, Israel

2 Baruch College and the Graduate Center of the City University of New York, New York, NY, USA when in control, participants would select locations that would maximize performance, based on metacognitive knowledge of structured memory representations. Although it is known that individuals possess knowledge of the content and workings of their memory systems, a phenomenon known as metamemory (e.g., Suchow, Fougnie, \& Alvarez, 2017), the ability to apply this knowledge in choosing one's own representations, remains unexplored.

In a recent study, we demonstrated the concept of SI WM using a modified spatial span task, in which participants constructed the spatial sequences they memorized (Magen \& Emmanouil, 2017). Participants constructed structured representations that benefited memory and flexibly manipulated these structures when asked to enhance or disrupt performance, revealing to some degree explicit metacognitive knowledge. Furthermore, self-initiation benefited memory beyond that of structure, suggesting that SI WM engages additional mechanisms.

The present study tested a novel SI spatial precision WM task, in which participants retained and reproduced single 
spatial locations they selected themselves. Spatial precision memory differs from memory for spatial sequences in terms of formed memory representations and processes, and therefore our predictions for the current study were derived from the literature on (provided) spatial precision WM (e.g., Huttenlocher, Hedges, \& Duncan, 1991). Memory for precise location information is often explored using the dot-location task, in which participants encode the location of a single dot presented within a bounded space, and reproduce the encoded location as accurately as possible after a short delay. Typically, the estimation errors show a systematic bias toward a prototypical area (typically the center) within the bounded space (e.g., Huttenlocher et al., 1991; Spencer \& Hund, 2002; Verbeek \& Spetch, 2008).

The category adjustment model (Crawford, Huttenlocher, \& Engebretson, 2000; Huttenlocher, Hedges, \& Veva, 2000) accounts for the observed estimation biases by assuming that spatial memory is hierarchical (Huttenlocher et al., 1991; Huttenlocher et al., 2000). Spatial locations are thought to be represented by two codes, a fine-grain location representation (which is inherently inaccurate), and a coarse representation of the spatial category to which the location belongs. Reproduction of the memorized location is a Bayesian combination of the two sources of information, leading to a biased estimation of the original location toward the spatial category prototype. The fine-grain location information by itself is unbiased, and is combined with the categorical information during estimation (Holden, Newcombe, \& Shipley, 2015; Huttenlocher et al., 1991; Sampaio \& Wang, 2012). Categories are typically defined based on prior knowledge or experience.

The biased estimations benefit memory performance by decreasing the overall estimation error (Huttenlocher et al., 1991; Huttenlocher et al., 2000). Indeed, the less reliable the finegrain information, the greater the categorical bias (i.e., reflecting more reliance on categorical information), with maximal bias when the fine-grain information is absent (Holden et al., 2015; Huttenlocher et al., 1991). Nevertheless, individuals are unaware of the use of categorical information, or that their estimations are biased toward the category prototype (Huttenlocher et al., 2000).

The observed bias in spatial estimations indicates that finegrain location representations in memory are mostly imprecise. One aspect of the environment that improves precision is the presence of landmarks. Performance in the dot-location task improves when the target location is close to the circle circumference, or when lines are drawn within the circular space. When landmarks are present, the location estimations are biased toward the landmarks instead of the category prototype (Huttenlocher et al., 1991; Verbeek \& Spetch, 2008).

The task in the current study was a modified dot-location task, in which participants memorized precise spatial locations they selected themselves, or locations provided to them.
Overall, our primary focus in the study was the distribution of locations participants selected, and the precision of memory for self-initiated compared to provided locations. Given the assumption that participants are unaware of the role of spatial categories in spatial estimation (Huttenlocher et al., 2000), we hypothesized that they may use landmarks in the visual scene (i.e., the screen edges) to improve performance. Furthermore, we hypothesized that SI encoding by itself would benefit performance (cf. Magen \& Emmanouil, 2017).

\section{Experiment 1}

Experiment 1 explored the strategies participants use when selecting to-be-remembered single locations, and how readily these strategies can be manipulated. In Experiment 1a, participants memorized a single location they selected themselves (SI condition), or a random computer-generated location provided to them (non-SI condition). The non-SI condition consisted of randomly distributed locations to avoid providing participants hints on potential strategies from nonrandom displays. To create a demanding task, selections were restricted to a square space that appeared only during encoding, positioned in random locations across the screen (i.e., to preclude trivial selections such as the corner of the screen). Consequently, participants were required to memorize precise spatial locations in different areas across the screen. Following a short delay, participants reproduced the locations they memorized as accurately as possible. We explored the distribution of the locations participants selected, and the estimation errors in the SI and the non-SI conditions.

In Experiment 1b, a new group of participants were presented with a similar memory task, but were asked to select locations that would disrupt memory performance for a hypothetical competitor (a condition termed the competition condition). We assumed that if the locations participants were to select in Experiment 1a were intended to enhance memory performance, then participants in Experiment $1 \mathrm{~b}$ would avoid selecting these locations. If, on the other hand, the locations participants were to select in Experiment 1a were unrelated to memory, but were, for instance, easier to select than others, then we would expect similar location distributions in Experiments 1a and $1 \mathrm{~b}$.

\section{Method}

Participants Forty-eight students from the Hebrew University participated in a 1-hour session (24 in each experiment). They provided informed consent before participating in the study for course credit or payment.

Stimuli, design, and procedure Participants sat in a dimly lit room at a distance of $100 \mathrm{~cm}$ from a 17 -inch CRT display, and 
rested their head on a chin rest. Each trial began with the presentation of a gray square area $\left(2.56^{\circ} \times 2.56^{\circ}\right.$ of visual angle) located in random locations across the screen, and the mouse cursor, which appeared at the screen center. The selection area could appear anywhere on the screen with the restriction that it did not overlap with a $3.26^{\circ} \times 3.26^{\circ}$ of visual angle area at the screen center, where the fixation cue and the mouse cursor appeared.

In the SI and competition conditions, participants selected a single location within the selection area by clicking on it with the left key of the mouse (see Fig. 1). A black square appeared in the selected location $\left(0.22^{\circ} \times 0.22^{\circ}\right.$ of visual angle $)$ and remained on the screen for $1,000 \mathrm{~ms}$. In the SI condition, participants were asked to select locations that would enhance their memory performance. In the competition condition, participants experienced the SI task for four trials and were then asked to select locations for a hypothetical competitor in a memory contest.

In the non-SI condition, the presentation of the selection area was followed after $500 \mathrm{~ms}$, by the presentation of a black square outline $\left(0.22^{\circ} \times 0.22^{\circ}\right.$ of visual angle), randomly located within the selection area, which marked the to-beremembered location. To equate the motoric responses in the SI and non-SI conditions, participants in non-SI trials clicked the marked location, turning the outline into a black square, which remained on the screen for 1,000 ms. Encoding was self-paced in all conditions. The mouse cursor disappeared after the participant clicked the chosen location and reappeared only during retrieval.

Following the encoding phase, a central fixation point appeared during a 2,000 ms delay and was replaced by the mouse cursor during retrieval. Participants reproduced the location, they or the computer selected, as accurately as possible by clicking on a location on the (blank) screen. Estimation errors were defined as the distance between the locations that were selected during encoding and retrieval. There were 120 trials in each of the SI, non-SI, and the competition encoding conditions. Participants received a break every 30 trials. The encoding manipulation in Experiment 1a was blocked and order was counterbalanced across participants. Each task in Experiments 1a and $1 \mathrm{~b}$ began with four practice trials.

\section{Results and discussion}

In all the experiments, errors larger than $2.56^{\circ}$ of visual angle (the size of the selection area), and RTs larger than three standard deviation from the mean, were removed from further analysis, accounting for less than $2 \%$ of the trials in all the experiments.

Experiment 1a: Encoding-Locations distribution To examine the participants' strategies, we first plotted the selected locations relative to the entire screen, which revealed similar distributions of locations in the SI and non-SI conditions (see Supplementary Materials, Fig. S1, for the results of all the experiments). Next, we plotted the selected locations relative to the square selection area. Given the assumption that participants may use landmarks across the screen, which are positioned in different relative positions in its four quadrants, the data were analyzed separately for each quadrant. As illustrated in Fig. 2a, while the locations in the non-SI task were distributed evenly across the selection area, in the SI condition participants most frequently selected locations at the center, followed by a preference for locations in the direction of the center of the screen, and locations closest to the corner of the screen.

To quantify the differences between the distributions, the selection area was divided into a $3 \times 3$ grid (each cell measuring approximately $0.85^{\circ} \times 0.85^{\circ}$ of visual angle), and the percentage of locations placed at each cell were calculated. The results were evaluated in a repeated-measures ANOVA with encoding condition (SI or non-SI), quadrants, and grid

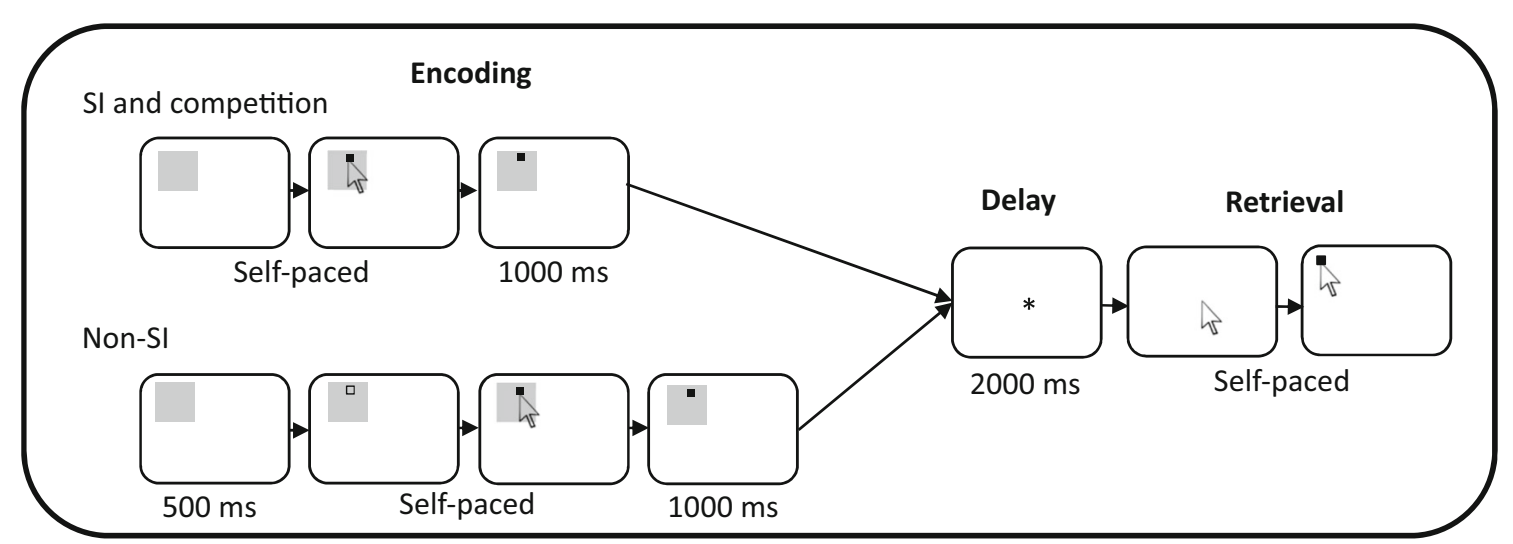

Fig. 1 Example of the trials in Experiments 1 and 2. During encoding, participants clicked on one location selected by them (SI or the competition conditions) or by the computer (non-SI condition). During retrieval, participants were presented with the mouse cursor at the center of a blank screen and were asked to reproduce the locations they encoded as accurately as possible. Note that the size of the selection area is not to scale for presentation purposes. SI = self-initiated 
a
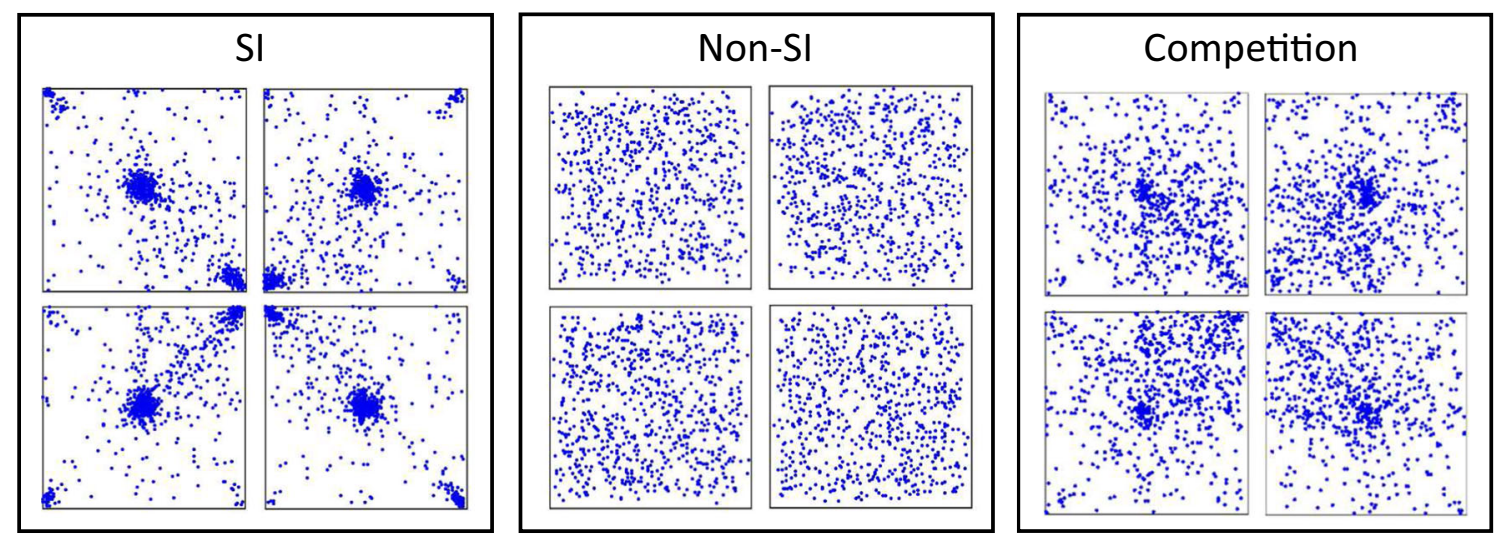

b
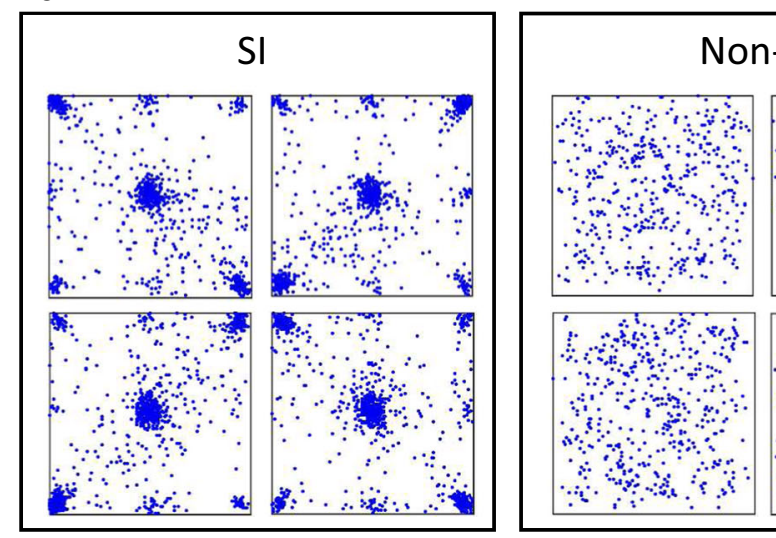

Fig. 2 The distribution of the locations of all participants, plotted relative to the selection area, presented separately for each quadrant. a Experiment 1. Left: Locations selected by participants (SI encoding condition). Center: Locations randomly selected by the computer (non-SI condition). Right: Locations selected by participants in the competition condition. b Experiment 2. Left: Locations selected by participants (SI

cells as factors (see Table 1 for statistics). The ANOVA revealed a significant main effect of cells, and significant interactions of encoding and cells, quadrants and cells, and a significant three-way interaction between encoding, quadrants, and cells.

We conducted an additional analysis using a KolmogorovSmirnov test (Fasano \& Franceschini, 1987) to verify the differences in the two-dimensional distributions of the SI and non-SI conditions. The difference was significant for 21 of the 24 participants, all significant $p$ s $<.009$.

The location distribution in the SI condition revealed the strategies participants employed during encoding. In all four quadrants participants selected the three areas running along the diagonal from the corner of the selection area closest to the screen corner, to the corner which was closest to the screen center. The percentage of trials in these cells are presented in Table 2. One-sample $t$ tests examined for each condition and quadrant, whether the percentage of locations in these cells exceeded the expected $33 \%$ of a random distribution. The
Bonferroni correction for multiple comparisons was used with a threshold of 0.0063 ( $0.05 / 8$ comparisons). The percentage of trials exceeded the expected $33 \%$ in the SI, but not in the nonSI condition. Thus, unlike our predictions, participants did not rely on landmarks such as the border of the selection area, which could have allowed them to calculate the absolute distance from the edge of the screen. Instead, they selected central locations that were necessarily less accurate. While the preference for the three cells along the diagonal was highly significant, there was also some variability between participants (see Supplementary Materials, Fig. S2).

Reaction time (RT) during encoding We compared encoding $\mathrm{RT}$ in the SI and non-SI conditions, although they represented different processes. RT was measured from the onset of the selection area and until the participants clicked the selected location. As seen in Figure 3a, RT was the same in the two encoding conditions and across quadrants, as none of the effects were significant (see Table 3 for statistics). 
Table 1. Univariate inferential statistics for the analysis of the locations distribution in Experiments 1a and 1b

\begin{tabular}{|c|c|c|c|c|c|c|}
\hline \multirow[b]{3}{*}{ Variable } & \multicolumn{2}{|c|}{ Experiment 1a } & \multicolumn{4}{|c|}{ Experiment 1a vs. Experiment 1b } \\
\hline & \multicolumn{2}{|c|}{ SI vs. non-SI } & \multicolumn{2}{|c|}{ SI vs. competition } & \multicolumn{2}{|c|}{ Non-SI vs. competition } \\
\hline & $F$ & $\eta_{\mathrm{p}}^{2}$ & $F$ & $\eta_{\mathrm{p}}^{2}$ & $F$ & $\eta_{\mathrm{p}}^{2}$ \\
\hline Cells & $21.60^{\mathrm{a}}$ & $0.484^{* * *}$ & $29.75^{\mathrm{c}}$ & $0.393^{* * *}$ & $14.55^{\mathrm{c}}$ & $0.240^{* * *}$ \\
\hline Encoding $\times$ Cells & $18.53^{\mathrm{a}}$ & $0.446^{* * * *}$ & $6.17^{\mathrm{c}}$ & $0.118^{* * * * *}$ & $5.86^{\mathrm{c}}$ & $0.113^{* * * *}$ \\
\hline Quadrants $\times$ Cells & $6.10^{\mathrm{b}}$ & $0.209^{* * * *}$ & $14.27^{\mathrm{d}}$ & $0.237^{* * *}$ & $6.31^{\mathrm{d}}$ & $0.121^{* * *}$ \\
\hline $\begin{array}{l}\text { Encoding } \times \text { Quadrants } \\
\times \text { Cells }\end{array}$ & $5.86^{\mathrm{b}}$ & $0.203^{* * * *}$ & $1.16^{\mathrm{d}}$ & 0.025 & $6.60^{\mathrm{d}}$ & $0.126^{* * *}$ \\
\hline
\end{tabular}

Note. SI = self-initiated. Note that the main effects of encoding and quadrants were zero, since they represent the overall number of trials, which was the same in the SI and non-SI conditions, and across quadrants

${ }^{\mathrm{a}} d f=1,84{ }^{\mathrm{b}} d f=24,255^{\mathrm{c}} d f=8,368^{\mathrm{d}} d f=24,1104$

$* * p<.01 . * * * p<.001$

\section{Retrieval}

Errors Estimation errors were defined as the distance in visual angle between the locations selected during encoding and retrieval (see Fig. 3a). The distribution of the responses relative to the selection area are presented in the Supplementary Materials (Fig. S3). Errors were significantly smaller in the SI condition in all four quadrants, as indicated by the significant main effect of encoding (see Table 4 for statistics). The main effect of quadrants was also significant. A series of follow-up $t$ tests explored the differences between the four quadrants (averaged across the two encoding conditions). The Bonferroni correction for multiple comparisons was used with a threshold of $p<.0063(0.05 / 8)$. The only significant differences were smaller errors in all quadrants relative to the bottom left quadrant (all $p$ s <.004).
Experiment $1 \mathrm{~b}$ :Encoding一Locations distribution The distribution of locations in Experiment $1 \mathrm{~b}$ was less organized than in the SI condition in Experiment 1a (see Fig. 2a), yielding a significant interaction between the cells and encoding effects. Nevertheless, this distribution was not random as was the distribution in the non-SI condition, revealing significant interactions between cells and encoding, and between cells, encoding, and quadrant (see Table 1 and Supplementary Materials Fig. S2).

Focusing on the three cells along the diagonal revealed that in the competition condition the percentage of locations in these cells exceeded the expected $33 \%$ of a random distribution (see Table 2; all the effects were significant given a corrected threshold of $0.0125=(0.05 / 4$ comparisons $))$. Nevertheless, the percentage of trials in these cells was significantly lower than in the SI condition. A repeated-measures ANOVA with quadrant

Table 2. Percentage of selected locations in the three cells running along the diagonal from the corner of the selection area closest to the screen corner, through the center of the selection area, and toward its corner which was closest to the screen center

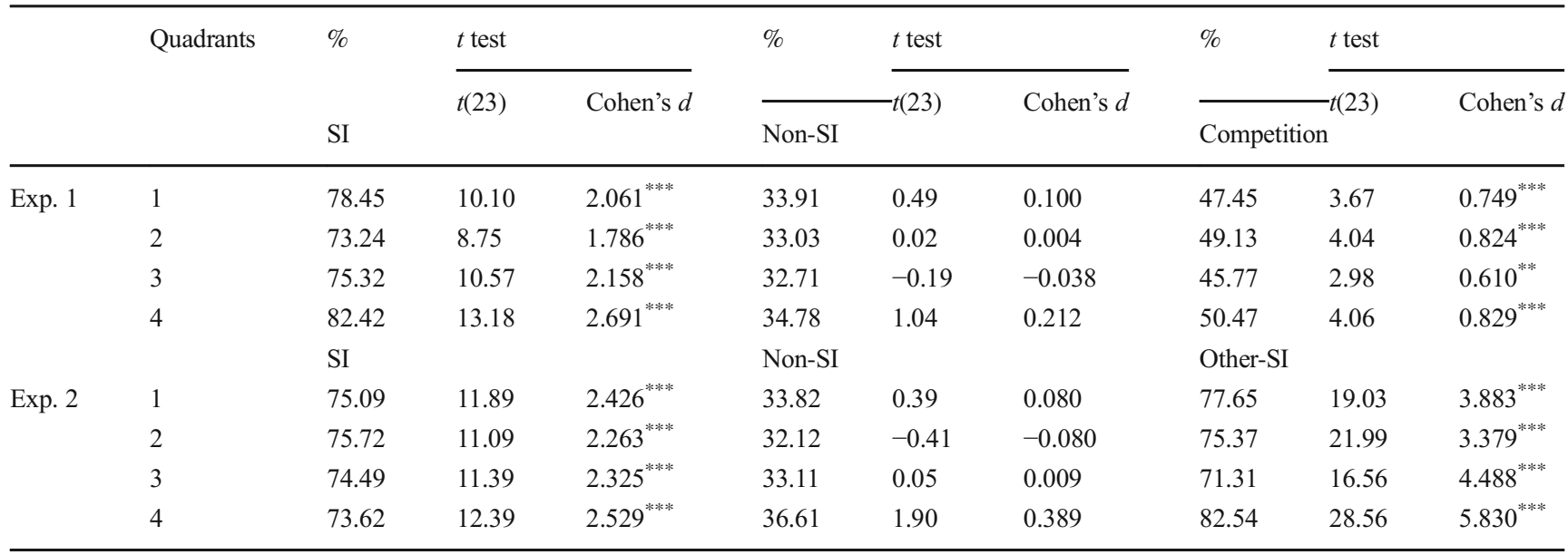

Note. Results are presented for each quadrant and each encoding condition in Experiments 1-2. One-sample $t$ tests explored whether the percentage of trials in these cells exceeded 33\%, the expected percentage in a random distribution. Quadrants are numbered from the top left and clockwise. $* * p<.01$. $* * * p<.001 . \mathrm{SI}=$ self-initiated 


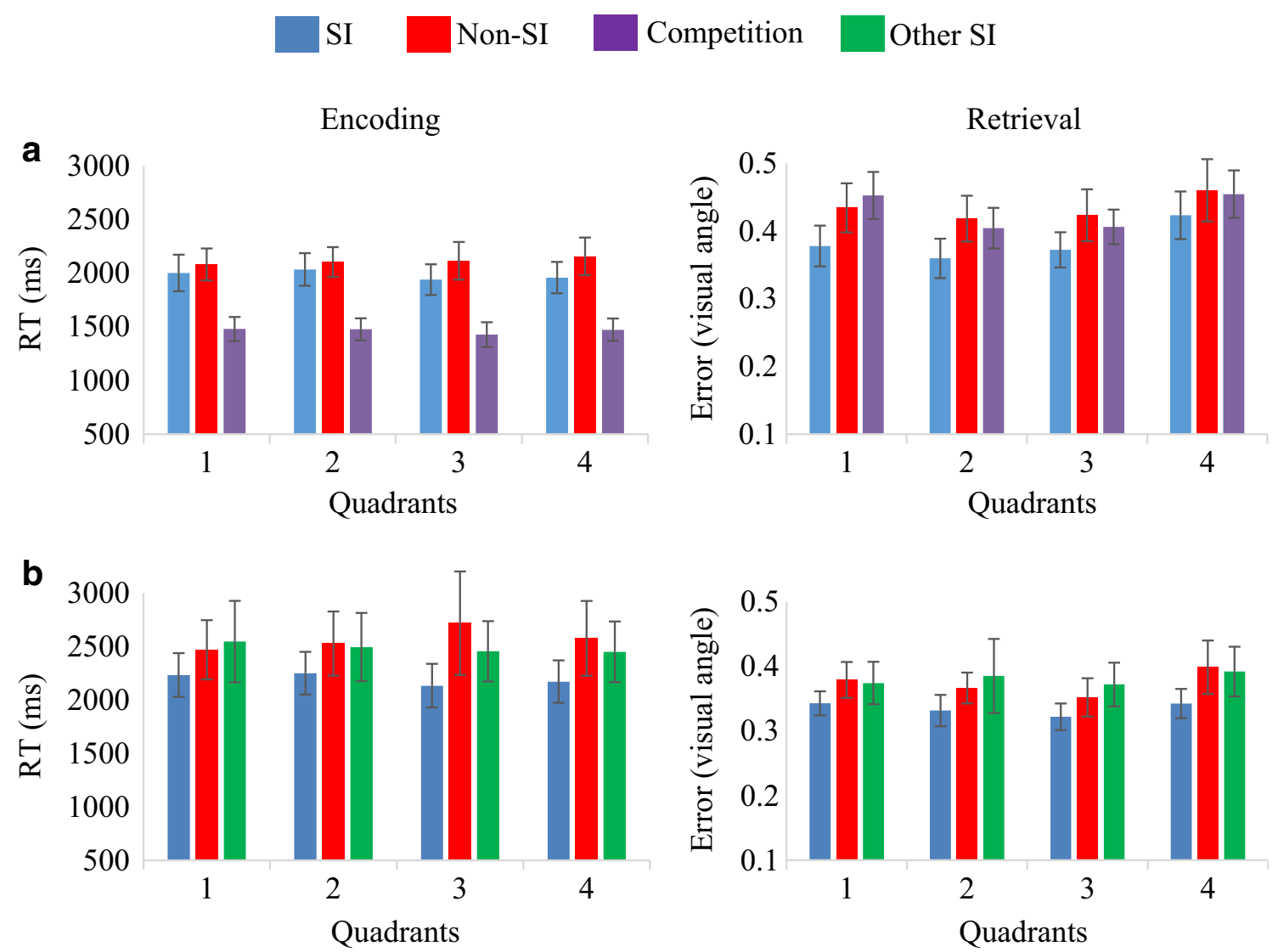

Fig. 3 Encoding RT (ms) (left) and errors (in visual angle) during retrieval (right) for the different encoding conditions in (a) Experiments 1a and 1b. b Experiment 2. Quadrants are numbered from the top left quadrant and clockwise. (Color figure online)

as a within-participant effect and encoding as a betweenparticipant effect showed a significant difference between the competition and the SI condition, $F(1,46)=28.14, p<.001$, $\eta_{\mathrm{p}}{ }^{2}=0.380$. Thus, participants in the competition condition avoided the prototypical locations across the diagonal, relative to the SI condition in Experiment 1a.

RT during encoding RT in the competition condition is presented in Fig. 3a. RT in this condition was significantly faster than in the SI or the non-SI conditions (see Table 4 for statistics), demonstrating that strategically selecting locations in an organized fashion is more demanding. This finding echoes the results of SI WM for spatial sequences (Magen \& Emmanouil, 2017).

Retrieval: Errors The estimation errors in the competition condition were intermediate between the SI and non-SI conditions of Experiment 1a, but were not significantly different from either condition (see Fig. 3a, Table 4 for statistics).

Overall, Experiment 1 demonstrated that when facing the decision to choose a to-be-memorized location, participants rely on available spatial categories (i.e., the selection

Table 3. Univariate inferential statistics for the analysis of the reaction time in Experiments $1 \mathrm{a}$ and $1 \mathrm{~b}$

\begin{tabular}{|c|c|c|c|c|c|c|}
\hline \multirow[b]{3}{*}{ Variable } & \multirow{2}{*}{\multicolumn{2}{|c|}{$\frac{\text { Experiment 1a }}{\text { Si vs. non-SI }}$}} & \multicolumn{4}{|c|}{ Experiment 1a vs. Experiment 1b } \\
\hline & & & \multicolumn{2}{|c|}{ SI vs. competition } & \multicolumn{2}{|c|}{ Non-SI vs. competition } \\
\hline & $F$ & $\eta_{\mathrm{p}}^{2}$ & $F$ & $\eta_{\mathrm{p}}^{2}$ & $F$ & $\eta_{\mathrm{p}}^{2}$ \\
\hline Encoding & $<1^{\mathrm{a}}$ & 0.040 & $7.98^{\mathrm{c}}$ & $0.148^{* *}$ & $11.58^{\mathrm{c}}$ & $0.201^{* * * *}$ \\
\hline Quadrants & $<1^{\mathrm{b}}$ & 0.024 & $2.04^{\mathrm{d}}$ & 0.043 & $<1^{\mathrm{d}}$ & 0.019 \\
\hline Encoding $\times$ Quadrants & $1.86^{\mathrm{b}}$ & 0.075 & $1.16^{\mathrm{d}}$ & 0.010 & $1.16^{\mathrm{d}}$ & 0.025 \\
\hline
\end{tabular}

${ }^{\mathrm{a}} d f=1,23^{\mathrm{b}} d f=3,69^{\mathrm{c}} d f=1,46^{\mathrm{d}} d f=3,138,{ }^{*} p<.01 . * * * p<.001$, SI $=$ self-initiated 
Table 4. Univariate inferential statistics for the analysis of errors in Experiments 1a and $1 \mathrm{~b}$

\begin{tabular}{|c|c|c|c|c|c|c|}
\hline \multirow[b]{3}{*}{ Variable } & \multicolumn{2}{|c|}{ Experiment 1a } & \multicolumn{4}{|c|}{ Experiment 1a vs. Experiment 1b } \\
\hline & \multicolumn{2}{|c|}{ Si vs. non-SI } & \multicolumn{2}{|c|}{ SI vs. competition } & \multicolumn{2}{|c|}{ Non-SI vs. competition } \\
\hline & $F$ & $\eta_{p}^{2}$ & $F$ & $\eta_{p}^{2}$ & $F$ & $\eta_{p}^{2}$ \\
\hline Encoding & $5.49^{\mathrm{a}}$ & $0.193^{*}$ & $1.28^{\mathrm{c}}$ & 0.027 & $<1^{\mathrm{c}}$ & 0.000 \\
\hline Quadrants & $7.00^{\mathrm{b}}$ & $0.233^{\text {**** }}$ & $8.33^{\mathrm{d}}$ & $0.153^{* * * *}$ & $5.39^{\mathrm{d}}$ & $0.105^{* *}$ \\
\hline Encoding $\times$ Quadrants & $<1^{\mathrm{b}}$ & 0.015 & $1.21^{\mathrm{d}}$ & 0.026 & $<1^{\mathrm{d}}$ & 0.015 \\
\hline
\end{tabular}

${ }^{a} d f=1,23^{b} d f=3,69^{c} d f=1,46^{d} d f=3,138 . * * p<.01 . * * * p<.001$. SI $=$ self-initiated

area and the entire screen) and adjust their selections toward prototypical areas within these categories. When asked to disrupt memory performance, participants avoid these very same prototypical locations, supporting the assumption that these locations were selected in the SI condition to enhance memory performance. Furthermore, memory for SI locations was more precise than memory for provided randomly distributed locations. It is unclear, however, from Experiment 1 whether the benefit was the result of the more organized location distributions in the SI condition, of selfinitiated encoding, or of both. This question was addressed in Experiment 2.

\section{Experiment 2}

In Experiment 2, participants performed the same WM task under three conditions. Two of them, the SI and non-SI conditions, were identical to those of Experiment 1a. In an additional third condition, termed the "other-SI" condition, participants memorized organized provided locations that were selected by participants in the SI condition in Experiment 1a. The other-SI condition was similar to a double-pass paradigm, in which participants respond to the same stimuli under different conditions, (e.g., Suchow et al., 2017). The location distributions in the other-SI condition reflected the sum of strategies that participants used to select the to-be-memorized locations, representing location distributions that individuals may encounter in their everyday surroundings, which are shaped by them or by others. We were interested to see whether these provided locations would benefit memory performance. Nevertheless, due to individual variability, we also compared memory performance in the SI and the provided conditions in specific locations that matched each participant's individual strategy.

If memory benefited from the spatial distribution of selected locations and not only from self-initiation, accuracy in the other-SI condition (or the matched provided condition) should be lower than in the SI condition, but superior relative to the non-SI condition (or the nonmatched provided condition).

\section{Method}

Participants Twenty-four students from the same pool as in Experiment 1 participated in this study in a 1-hour session, all provided informed consent before participating in the study.

The method was identical to Experiment 1, except for the following. The provided trials were divided equally between the non-SI and the other-SI conditions, which were selected randomly from the non-SI and the SI conditions in Experiment 1a, respectively. The SI and provided (non-SI and other-SI) conditions were presented in separate blocks, with 160 trials in each block. Participants received a break every 40 trials. Block order was counterbalanced across participants, and each block began with four practice trials.

\section{Results and discussion}

The same analyses as in Experiment 1 were conducted in Experiment 2.

Encoding: Locations distribution The location distributions were analyzed in each quadrant relative to the selection area (see Fig. 2b and Table 2; individual data are presented in Supplementary Materials, Fig. S4). The SI condition was similar to the SI condition in Experiment 1a, showing a preference for locations at the center of the selection area, and in its corners, which were in the direction of the center and the corner of the entire screen. Unsurprisingly, the other-SI and the non-SI conditions were similar to the SI and non-SI conditions in Experiment 1a, respectively. A repeated-measures ANOVA revealed that all the main effects and interactions between encoding, cells, and quadrants were significant (see Table 5 for statistics). Follow-up ANOVAs revealed significant interactions between pairs of encoding conditions; the location distribution in the SI condition differed significantly from both the other-SI and the non-SI conditions. The significant difference between the SI and other-SI conditions reflected variability between participants, as the location distributions in both conditions reflected similar strategies. As shown in Table 2, focusing the analysis on the three cells along the diagonal showed 
Table 5. Univariate inferential statistics for the analysis of the locations distribution in Experiment 2

\begin{tabular}{llll}
\hline Variable & $d f$ & $F$ & $\eta_{\mathrm{p}}{ }^{2}$ \\
\hline Overall analysis & & & \\
$\quad$ Cells & 8,184 & 65.87 & $0.741^{\text {**** }}$ \\
Encoding $\times$ Cells & 16,368 & 14.39 & $0.385^{\text {**** }}$ \\
Quadrants $\times$ Cells & 24,552 & 10.97 & $0.323^{\text {**** }}$ \\
Encoding $\times$ Quadrants $\times$ Cells & 48,1104 & 5.53 & $0.194^{* * * *}$ \\
SI vs. Other-SI & & & \\
Cells & 8,184 & 62.97 & $0.732^{\text {**** }}$ \\
Encoding $\times$ Cells & 8,184 & 3.42 & $0.129^{\text {**** }}$ \\
Quadrants $\times$ Cells & 24,552 & 13.49 & $0.370^{\text {**** }}$ \\
Encoding $\times$ Quadrants $\times$ Cells & 24,552 & 3.28 & $0.125^{\text {**** }}$ \\
SI vs. Non-SI & & & \\
Cells & 8,184 & 13.64 & $0.372^{\text {**** }}$ \\
Encoding $\times$ Cells & 8,184 & 9.56 & $0.293^{* * * *}$ \\
Quadrants $\times$ Cells & 24,552 & 4.18 & $0.154^{* * * *}$ \\
Encoding $\times$ Quadrants $\times$ Cells & 24,552 & 4.59 & $0.166^{* * * *}$ \\
\hline
\end{tabular}

$* * p<.01 . * * * p<.001$. SI $=$ self-initiated. Note that the main effects of encoding and quadrants were zero, since they represent the overall number of trials, which was the same in the SI and non-SI conditions, and across quadrants

significant deviations from random selections in the SI and other-SI conditions, but not in the non-SI condition (corrected threshold, $0.0042(0.05 / 12)$.

A Kolmogorov-Smirnov test (Fasano \& Franceschini, 1987) that compared the SI to the non-SI conditions was significant for all participants, all $p \mathrm{~s}<.02$.

RT during encoding Although encoding RT varied numerically across the three encoding conditions (see Fig. 3b), the main effect of encoding was nonsignificant, $F(2,46)=1.07$, neither were the main effect of quadrants and its interaction with encoding $F(3,69)<1$ and $F(6,138)<1$, respectively. Planned contrasts comparing the SI condition to the non-SI and the other-SI conditions were nonsignificant as well $F(1$, $23)=1.22$ and $F(1,23)<1$, respectively.

Retrieval: Errors As illustrated in Fig. 3b, estimation errors were smallest in the SI condition and similar in the non-SI and the other-SI conditions. A repeated-measures ANOVA showed a significant main effect of encoding $F(2,46)=$ $3.44, p<.05, \eta_{\mathrm{p}}{ }^{2}=0.130$, and nonsignificant effects of quadrants $F(3,69)=1.49, p=.23, \eta_{\mathrm{p}}{ }^{2}=0.061$, or of its interaction with encoding $F(6,138)<1$. Planned contrasts revealed a significant difference between the SI and the non-SI conditions, $F(1,23)=6.45, p<.05, \eta_{\mathrm{p}}{ }^{2}=0.219$, and a marginally significant difference between the SI and the other-SI conditions $F(1,23)=3.81, p=.06, \eta_{\mathrm{p}}^{2}=0.142$. A post hoc comparison of the non-SI and the other-SI conditions was nonsignificant, $p=1$, although the location distributions in these two conditions varied greatly.

An additional analysis compared accuracy in the SI and the provided condition matched to the locations participants selected in the SI condition (i.e., reflecting individual strategies). The analysis matched locations by cells, and for each participant included only cells in which more than $10 \%$ of the locations were placed in the SI condition (these ranged between one and five cells across participants, with an average of 3.21 cells). We assumed that cells in which less than $10 \%$ of the locations were placed represented random placements. In the analysis, the number of trials were equated across the encoding conditions. For each cell, the minimum number of trials (from either the SI or provided conditions) was used, and a random subset of trials was selected from the other condition that contained more trials than this minimum. The analysis revealed significantly smaller errors in the SI condition relative to the matched provided condition $\left(M=0.34^{\circ}\right.$ and $0.38^{\circ}$, respectively), $t(23)=1.90, p<.05$, Cohen's $d=0.39,\left(M_{\text {number }}\right.$ of trials $=64.58)$. Next, accuracy in the matched and nonmatched provided conditions was compared to explore whether the strategic locations by themselves benefited memory performance. Here as well, the number of trials were equated in the two conditions based on the minimum number of trials in either condition, and a random subset of trials was selected from the condition that contained more trials than this minimum. The analysis yielded similar accuracy levels in the matched and nonmatched provided conditions $\left(M=0.38^{\circ}\right.$ and $0.37^{\circ}$, respectively), $t(23)=0.35, p=.73$, Cohen's $d=0.07$ $\left(M_{\text {number of trials }}=56.46\right)$.

The results of Experiment 2 demonstrated that the enhanced memory performance in the SI encoding condition was solely the result of maintaining self-initiated locations. One explanation for this result may be the absence of the selection area during retrieval, the space in which the locations were encoded. To explore this possibility, we ran an experiment identical to Experiment 2, except that the selection area reappeared during retrieval $(n=24)$. The results revealed very small errors, especially in the SI condition. Similar to Experiment 2, the analysis of errors revealed a significant difference between the SI and the matched provided condition, $\left(M=0.11^{\circ}\right.$ and $0.19^{\circ}$, respectively), $t(23)=5.25, p<$ .001 , Cohen's $d=1.07,\left(M_{\text {number of trials }}=60.29\right)$. Unlike Experiment 2, a significant difference was also found between the matched and nonmatched provided conditions $\left(M=0.19^{\circ}\right.$ and $0.22^{\circ}$, respectively), $t(23)=2.07, p<.05$, Cohen's $d=$ $0.50,\left(M_{\text {number of trials }}=47.21\right)$. These findings may suggest that the locations participants selected in Experiment 2 were considered only relative to the encoding and not the retrieval context (i.e., relative to the selection area, which did not reappear during retrieval). However, the strategies participants used to select the to-be-memorized locations were somewhat different when the selection area reappeared during retrieval. 
Specifically, more emphasis was put on the corners of the selection area (see Supplementary Materials, Fig. S5). Thus, the SI encoding strategies in Experiment 2 were, to some extent, also influenced by the absence of the selection area during retrieval.

\section{General discussion}

The current study takes an important step in understanding the mechanisms of self-initiated WM, a prevalent aspect of everyday behavior. In a modified spatial WM task, participants marked a location within a square selection area that randomly appeared across the screen. In two experiments we tested the distribution of locations that participants chose when asked to enhance or disrupt memory performance, as well as their accuracy in the SI and provided conditions. The results showed that participants' selections were organized with a preference for the center of the selection area or its corners that were closest to the center or the corners of the entire screen. Selections of these locations were less frequent when participants attempted to disrupt memory performance. Furthermore, estimation errors were smaller for the locations participants selected compared to provided locations, even when the self-initiated and the provided locations were similar in their distribution. These findings suggest that SI WM was facilitated for reasons other than their location distribution and suggest that the resources at the disposal of SI WM exceed those of WM for provided information.

We interpret the results within the framework of the category adjustment models (Crawford et al., 2000; Huttenlocher et al., 2000). When actively selecting a location to encode in memory, participants resort to choosing prototypical locations of perceived spatial categories in the visual scene (i.e., the selection area and the entire screen) in an attempt to minimize their overall error. The results suggest that participants have access to sophisticated metacognitive knowledge about the utility of categorical information during the reconstruction of memorized spatial representations. Nevertheless, when the selection area was absent during retrieval, the selected locations in the SI condition had no impact on accuracy, which was solely determined by self-initiation.

One possible interpretation of this finding is that although participants strategically selected locations with the intention to improve memory performance, they did not have access to the appropriate metacognitive knowledge. This conclusion, however, stands in contrast with the literature on the dotlocation task, which emphasizes the important role in memory of the very same locations that participants selected (Huttenlocher et al., 1991). Alternatively, the absence of the selection area during retrieval, which provided the spatial context during encoding, may have prevented participants from benefiting from the locations they selected.
Indeed, when the selection area reappeared during retrieval, the locations participants selected benefited memory performance. However, the distribution of locations was somewhat different in this experiment. Thus, while participants in Experiments 1 and 2 were aware of the absence of the selection area during retrieval (as reflected in their strategies), they still, to a large extent, selected locations based on the context that was present only during encoding. These findings have important implications for the nature of SI WM. In real life, unlike more artificial laboratory tasks, the future context is not always predictable, and therefore participants may mostly consider their current state during encoding. If proven true in future studies, it points to a potential limitation in SI WM that has not been addressed in the literature thus far, and may impact everyday performance. The flexibility of SI encoding in light of transformed encoding and retrieval contexts, and their impact on memory performance should be clarified further in future studies.

Relatedly, even if participants mostly consider their current state during encoding, to perform efficiently they must be able to adjust their SI encoding strategies to adapt to changing environments. In provided spatial WM, the bias in location estimation tasks is adjusted continuously based on experience (Lipinski, Simmering, Johnson, \& Spencer, 2010). For example, prototypical locations differ as a function of the shape of the bounded space (Wedell, Fitting, \& Allen, 2007). Future studies should investigate how well participants adjust their SI encoding strategies in changing encoding environments.

Finally, SI WM showed an advantage, regardless of the locations participants selected. Several processes may have benefited memory performance. For instance, long-term memory performance is often enhanced for selfreferenced or self-generated information (Cunningham, Turk, Macdonald, \& Macrae, 2008; Slamecka \& Graf, 1978). Furthermore, the control participants had over the memory displays in the SI condition, may have increased their sense of agency during the task, which is known to have a beneficial impact on memory (e.g., Murty, DuBrow, \& Davachi, 2015).

Studies of WM thus far have failed to capture the complexity of people's interactions with the world, and the way they construct their surroundings to enhance memory performance. To do so, individuals must analyze their current and future surroundings, while integrating metacognitive knowledge regarding the strengths and weaknesses of their cognitive system. The processes involved in spatial and nonspatial SI WM and how efficiently participants can adapt their choices in changing environments are fruitful topics for future investigations.

Acknowledgements This research was supported by The Israel Science Foundation (Grant No. 771/12 to H.M.). 


\section{References}

Crawford, L. E., Huttenlocher, J., \& Engebretson, P. H. (2000). Category effects on estimates of stimuli: Perception or reconstruction. Psychological Science, 11, 280-284.

Cunningham, S. J., Turk, D. J., Macdonald, L. M., \& Macrae, C. N. (2008). Yours or mine? Ownership and memory. Consciousness and Cognition, 17, 312-318.

Fasano, G., \& Franceschini, A. (1987). A multidimensional version of the Kolmogorov-Smirnov test. Monthly Notices of the Royal Astronomical Society, 225, 155-170.

Holden, M. P., Newcombe, N. S., \& Shipley, T. F. (2015). Categorical biases in spatial memory: The role of certainty. Journal of Experimental Psychology: Learning, Memory, and Cognition, 41, 473-481.

Huttenlocher, J., Hedges, L. V., \& Duncan, S. (1991). Categories and particulars: Prototype effects in estimating spatial location. Psychological Review, 98, 352-376.

Huttenlocher, J., Hedges, L. V., \& Veva, J. L. (2000). Why do categories affect stimulus judgments? Journal of Experimental Psychology: General, 129, 220-241.

Lipinski, J., Simmering, V. R., Johnson, J. S., \& Spencer, J. P. (2010). The role of experience in location estimation: Target distributions shift location memory biases. Cognition, 115, 147-153.
Magen, H., \& Emmanouil, T. A. (2017). Working memory for selfinitiated and provided spatial configurations. Quarterly Journal of Experimental Psychology. https://doi.org/10.1177/ 1747021817739808

Murty, V. P., DuBrow, S., \& Davachi, L. (2015). The simple act of choosing influences declarative memory. Journal of Neuroscience, 35 , 6255-6264.

Sampaio, C., \& Wang, R. F. (2012). The temporal locus of the categorical bias in spatial memories. Journal of Cognitive Psychology, 24, 781788.

Slamecka, N. J., \& Graf, P. (1978). The generation effect: Delineation of a phenomenon. Journal of Experimental Psychology: Human Learning \& Memory, 4, 592-604.

Spencer, J. P., \& Hund, A. M. (2002). Prototypes and particulars: Geometric and experience-dependent spatial categories. Journal of Experimental Psychology: General, 131, 16-37.

Suchow, J. W., Fougnie, D., \& Alvarez, G. (2017). Looking inward and back: Real-time monitoring of visual working memories. Journal of Experimental Psychology: Learning, Memory and Cognition, 43, 660-668.

Verbeek, E., \& Spetch, M. (2008). Distortions in location memory. Psychonomic Bulletin \& Review, 15, 328-33.

Wedell, D. H., Fitting, S., \& Allen, G. L. (2007). Shape effects on memory for location. Psychonomic Bulletin \& Review, 14, 681-686. 